\title{
Process evaluation of a cluster randomised controlled trial of a school-based fruit and vegetable intervention: Project Tomato
}

\author{
Meaghan S Christian ${ }^{1, *}$, Charlotte EL Evans ${ }^{1}$, Joan K Ransley ${ }^{1}$, Darren C Greenwood ${ }^{2}$, \\ James D Thomas' and Janet E Cade ${ }^{1}$ \\ ${ }^{1}$ Nutritional Epidemiology Group, School of Food Science and Nutrition, University of Leeds, Leeds LS2 9JT, UK: \\ ${ }^{2}$ Centre for Epidemiology and Biostatistics, University of Leeds, Leeds, UK
}

Submitted 23 November 2010: Accepted 16 June 2011: First published online 11 August 2011

\begin{abstract}
Objective: The present analysis evaluates the overall appreciation and implementation of an intervention, Project Tomato, designed to maintain fruit and vegetable intake in children aged $8-9$ years.

Design: A random sample of fifty-four English primary schools (658 children) were randomised to either the intervention group or the control. The intervention group received a multi-component programme delivered in school by teachers and items sent home for parents/children. Dietary measurements were collected at baseline and follow-up. The intervention participants completed questionnaires on the intervention materials, to identify implementation and appreciation of the intervention, and other environmental mechanisms.

Setting: Fifty-four primary schools were randomly selected, with twenty-seven schools allocated to the intervention group.

Subjects: A total of 311 children received the intervention.

Results: Implementation of the intervention was low, $21 \cdot 3 \%$ of school items and $56.0 \%$ of home items were implemented. The intervention materials were well received by teachers, parents and children. Other mechanisms that may affect fruit and vegetable intake were explored. Children who ate their main meal with their parents 3-7 nights/week on average consumed 37.6 (95\% CI 9.8, 65.4) g more fruit and vegetables than children who ate with their parents 0-2 times/week.

Conclusions: Implementation of the trial components was poor. However, the results identified the importance of parental environment and mealtime structure on children's fruit and vegetable intake.
\end{abstract}

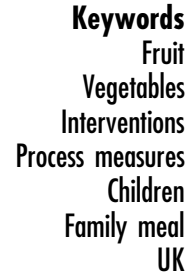

Children's health and nutritional intake remain a national priority in the UK. Nutritional education programmes have been developed for schools, home and community settings in an attempt to improve children's diets; however, it is still unclear which methodologies increase or maintain children's fruit and vegetable consumption.

As part of the UK's '5 A DAY' programme in 2004, the Department of Health launched the School Fruit and Vegetable Scheme (SFVS), aimed at increasing children's fruit and vegetable consumption by providing a free piece of fruit or vegetable every school day for children in Reception to Year 2 (aged 4-6 years). Three academic studies have evaluated the $\mathrm{SFVS}^{(1-3)}$, suggesting that while the scheme appears to increase children's fruit intake, this increase is only in the short term. When children are no longer eligible for the SFVS, fruit and vegetable intakes reduce to their original levels. The flexible multi-component intervention, Project Tomato, was designed to tackle this issue, aiming to encourage children to maintain fruit and vegetable consumption once eligibility for the SFVS ceased.

The Project Tomato intervention focused on curriculumbased activities and tasting sessions to improve children's knowledge and awareness of dietary fruit and vegetables. However, the home environment and mealtime structure are vital components of a child's eating pattern ${ }^{(4)}$. To tackle this issue, the Project Tomato intervention incorporated fun activities for parents and children to do together at home, because parents play a direct role in their child's eating behaviours and attitudes towards food $^{(4,5)}$.

Several systematic reviews have summarised existing research on school interventions to increase children's fruit and vegetable intake ${ }^{(6-8)}$ and these reviews suggest multi-component interventions are the most successful in terms of improving fruit and vegetable consumption ${ }^{(9-17)}$, supporting the design methodology used to create the Project Tomato intervention. Some of the successful studies 
that have previously used multi-component interventions included the following elements in their interventions:

1. integrating teaching about fruit and vegetables into the curriculum $^{(9)}$;

2. training of catering staff (verbal encouragement) ${ }^{(14)}$;

3. hands-on exposure (tasting and preparation sessions) ${ }^{(18)}$;

4. parental involvement through newsletters and homework activities ${ }^{(18,19)}$;

5. whole school approach (developing a nutrition policy, evening activities) ${ }^{(10-13)}$.

These elements were incorporated into the materials for the Project Tomato intervention. Further information about the theory behind the development of the Project Tomato intervention is described elsewhere ${ }^{(20)}$.

With such complex interventions, identifying the combination of components that are the most effective at increasing children's fruit and vegetable intake is difficult, illustrating the importance of exploring the process evaluations for each study. Process evaluations are used to improve the understanding of successful or unsuccessful health interventions; to identify the key components that make an intervention successful, for boys, girls or both; and to determine which environments/conditions lead to these particular components facilitating a successful outcome $^{(21,22)}$. Two terms commonly used in process evaluations and applied to the present study are implementation $^{(9,11-13,15)}$, meaning to what extent the intervention or intervention items were used by the intended audience; and appreciation ${ }^{(5,10,16,17,19,23)}$, a rating of how much the participants liked the intervention or intervention items $^{(22)}$. However, a vital component of process measures is to identify possible mediating variables. Several studies ${ }^{(5,14,16,19,23)}$ have gathered information from observations, interviews and questionnaires, and used multilevel regression analysis to take into consideration the nested design (children, within schools), to investigate the effect of the intervention on either implementation or appreciation of fruit and vegetable consumption. Some of the interesting associations with fruit and vegetable intake that have been identified are verbal encouragement by food staff and number of different types of fruit and vegetables available at lunch $^{(10)}$. While an intervention reported by Story et al. ${ }^{(23)}$ achieved a high implementation of school items, there were no differences in fruit and vegetable intake from children at schools with low implementation compared with high implementation. They did report that items which were less likely to be implemented were 'preparation intense' items such as the tasting activities.

In contrast, Wind et al. ${ }^{(5)}$ discovered that while the fruit and vegetable intake did not differ between intervention and control groups, the process evaluations revealed that the level of implementation and children's appreciation of the intervention were significantly associated with increase in fruit intake. Furthermore, Wind et al ${ }^{(5)}$ identified a trend in parental involvement, children with higher parental involvement on average had higher vegetable intake, although the difference was not statistically significant. Due to the nature of the school and home environment, different styles of intervention item are used in the different environmental conditions. Determining which environment intervention items aid change in fruit and vegetable consumption is essential for future research.

Despite the promising theory-based design of the Project Tomato intervention, it was not successful at maintaining children's fruit and vegetable intake (JK Ransley, MS Christian, CEL Evans et al., unpublished results). As stated in the protocol $^{(20)}$, the aim of the present study was to investigate the level of implementation and appreciation of the different elements involved in the Project Tomato intervention, to identify which elements worked and which did not, as well as explore other possible factors within the home environment that may be associated with fruit and vegetable intake.

\section{Methods}

\section{Population and study design}

A nationally representative sample of 1031 children from fifty-four schools was recruited into the trial ${ }^{(20)}$. Schools were randomised to receive the Project Tomato intervention or control. Further detail on the sampling methodology is reported elsewhere ${ }^{(20)}$. Ethical approval was obtained through the University of Leeds Central Research Ethics Committee.

\section{Process evaluation}

Twenty-four schools and 311 children completed the intervention. These schools were asked to complete a set of questionnaires on the intervention materials, sent at different periods during the intervention, for the teacher, parents or children. The teachers' questionnaire asked if they had completed each school item and to comment on a rating scale for each item. For example, teachers were asked to tick if they did or did not use the lesson plan, and then rate their appreciation of the lesson plan on a Likert scale from 1 to 5 . The parent questionnaire asked the parents to tick boxes if their child had received different intervention materials (yes or no), and to confirm if they had used these materials. Parents were also asked to comment on whether they had sent their child to school with fruit or vegetables, along with other family eating pattern questions; for example, how many nights a week they ate their main meal with their child at a table. How many days a week parents sent their children to school with a piece of fruit or vegetable was categorised into 0-1, 2-3, 4-5 per week, and how many nights a week they at their main meal together was categorised into 0-2 and 3-7 nights per week. The children were asked to complete three questionnaires. Each questionnaire asked the children to comment on the intervention items sent in the three kit bags, using a Likert scale, with the children 
asked to tick one of the following options: 'love it', 'like it', 'don't like it', 'hate it' or 'don't know'. The children were also asked to tick if they had or had not used each item.

\section{The intervention}

The intervention was designed using the theories of maintenance of health behaviour as a basis ${ }^{(24)}$. To apply the theories, underlying factors that have an effect on initiating and maintaining change in people's eating behaviour were described using the acronym FRAME: familiarisation, repetition, activities, modelling and environment ${ }^{(7,20,24)}$. The intervention consisted of core and customised materials designed for the whole school, the Year 3 classroom and for the children to take home. The core elements consisted of the following: the Project Tomato manual and twelve curriculum-related lesson plans. The customised elements were: cooking (designed by the British Nutrition Foundation); growing club information (designed by the Royal Horticultural Society); funding support; and information to set up a Project Tomato Team. The customised elements were tailored to meet each school's needs. The head teacher could decide which of these elements were included in his/her school's package. The intervention also involved items designed for the parents and children to complete, consisting of: three Project Tomato kit bags, two Project Tomato newsletters, and parent handouts. The duration of the intervention was 10 months from July 2007 to April 2008, and these materials were sent out at appropriate intervals throughout the school year.

\section{Dietary assessment}

Dietary intake was collected using a $24 \mathrm{~h}$ dietary assessment tool, the CADET (Child And Diet Evaluation Tool) diary $^{(25)}$. A National Foundation for Educational Research (NFER) field worker filled in the CADET diary for each child during school hours, and parents were asked to complete the evening and morning food consumption for their child.

\section{Statistical analyses}

To assess potential bias among parents and children who returned a process measures questionnaire and those who did not, a multilevel regression model was conducted on school and child characteristics, to explore if there were any differences between the two groups.

Descriptive statistics for implementation and appreciation of school and home items were calculated for teachers, children and parents. Participants' implementation and appreciation of either set of materials (school, home) were re-coded into variables with a range from 0 to 100 (0 meaning no items used or low implementation/ appreciation; 100 meaning all items used/implemented or high appreciation). The total scores for implementation of curriculum lessons and tasting sessions were categorised into binary codes: yes and no. The total scores for implementation of parents were coded into tertiles: low, medium and high. The total scores for appreciation for all teachers and parents were categorised into low and high.

To assess the association of different intervention materials (appreciation and implementation) on the difference in children's total fruit and vegetable intake, a two-level regression model was used with total weight of fruit and vegetables as the predictor. This model took into consideration the hierarchical structure of the data caused by randomising by cluster (school) ${ }^{(20)}$. Two models were assessed, the first was unadjusted and the second was adjusted for age, gender, ethnicity and Index of Multiple Deprivation (IMD) score ${ }^{(26)}$. The mean and 95\% confidence interval were generated for all models. To assess other potential mechanisms associated with total fruit and vegetable intake, a two-level regression model (unadjusted and adjusted) was used with total weight of fruit and vegetables as the predictor. All analyses were completed using the STATA statistical software package version $11 \cdot 2$ (StataCorp., College Station, TX, USA).

\section{Results}

The main outcome of the Project Tomato intervention has been reported elsewhere (JK Ransley, MS Christian, CEL Evans et al., unpublished results). Of the twenty-four schools that completed the interventions, 79\% (nineteen teachers) completed the teacher questionnaire. The number of children who participated in the intervention with a completed baseline and follow-up CADET food diary was 311. Of these children, 84\% (261) responded to one of the three children's questionnaires. Of parents, $38 \%$ (120) completed the parents' questionnaire.

The sensitivity analysis, conducted to determine differences between children who completed a process measure questionnaire and children who did not, revealed there were no differences for child or school characteristics (Table 1).

\section{Implementation and appreciation of the intervention for teachers}

Of the twenty-four intervention schools that completed the process measures questionnaires, eight schools did not implement any of the school items stated above. On average, schools implemented $21 \%$ of all school items. Table 2 describes the mean implementation and appreciation, and their 95\% confidence intervals, for the school items. School curriculum lessons and tasting sessions were the most widely implemented items, while the most appreciated items were the tasting sessions and the cooking club lessons.

\section{Implementation and appreciation of the intervention for parents and children}

The parents and children on average had low implementation scores of the home items, with the parents implementing 35\% (95\% CI 30, 40; $n$ 57) and the children implementing 56\% (95\% CI 53, 60; $n$ 115). Both parents' 
Table 1 Intervention demographic characteristics of the respondent and non-respondent participants: process measurement questionnaires

\begin{tabular}{lcccc}
\hline Characteristic & Completed a questionnaire & $95 \% \mathrm{Cl}$ & Did not complete a questionnaire & $95 \% \mathrm{Cl}$ \\
\hline Child characteristics & & & & $7 \cdot 0$ \\
$\quad$ Age at baseline (years) & $7 \cdot 0$ & $6 \cdot 9,7 \cdot 0$ & $56(28 / 50)$ & $6 \cdot 9,7 \cdot 1$ \\
\% Sex (boy) & $51(132 / 261)$ & 45,57 & $11(5 / 45)$ & 2,70 \\
$\%$ Ethnicity (non-white) & $13(33 / 246)$ & 9,18 & $24 \cdot 8$ & $23 \cdot 4,26 \cdot 3$ \\
Weight at baseline (kg) & $24 \cdot 6$ & $24 \cdot 1,25 \cdot 1$ & $123 \cdot 3$ & $121 \cdot 6,124 \cdot 9$ \\
Height at baseline (cm) & $122 \cdot 5$ & $121 \cdot 9,123 \cdot 1$ & $17 \cdot 6$ & 13,25 \\
School characteristics & $6 \cdot 6$ & 6,8 & $23 \cdot 8$ & $18 \cdot 9,30 \cdot 0$ \\
\% Free school meal eligibility & $13 \cdot 7$ & $12 \cdot 5,15 \cdot 1$ & & \\
IMD score & & & & \\
\hline
\end{tabular}

IMD, Index of Multiple Deprivation.

Completed a questionnaire ( $n$ 261); did not complete a questionnaire $(n 50)$.

Significant difference between groups: ${ }^{\star \star \star} P<0.001$ (\% free school meals eligibility and IMD score are transformed using natural log).

Table 2 Teacher's implementation and appreciation of the Project Tomato items (range 0-100)

\begin{tabular}{|c|c|c|c|c|}
\hline \multirow[b]{2}{*}{ Intervention item } & \multicolumn{2}{|c|}{ Implementation (\%) } & \multicolumn{2}{|c|}{ Appreciation (\%) } \\
\hline & Mean & $95 \% \mathrm{Cl}$ & Mean & $95 \% \mathrm{Cl}$ \\
\hline Curriculum lessons & 45 & 40,50 & 66 & 61,71 \\
\hline Tasting sessions & 25 & 21,30 & 86 & 83,87 \\
\hline Cooking club & 8 & 5,10 & 85 & 81,87 \\
\hline Gardening club & 8 & 6,11 & 47 & 42,51 \\
\hline All school intervention items $(n 24)$ & 21 & 19, 24 & 66 & 60,71 \\
\hline
\end{tabular}

and children's appreciation of the Project Tomato items was high, with a mean for parents of $76 \%(95 \%$ CI 73,$81 ; n 112)$ and for children of $73 \%$ (95\% CI 71, 76; $n$ 101). From the three child process measure questionnaires, the children were asked to comment if they used each item sent to them in one of the three kit bags. The items that were, on average, used the most were the fruit and veg portion game (178/ $221 ; 81 \%, 95 \%$ CI 75, 86), the fruit 'n' veg snack box for fruit (127/221; 58\%, $95 \%$ CI 50, 64), the Eat5! quiz book (97/181; $54 \%, 95 \%$ CI 46,61$)$ and the balance of good health quiz book (131/236; 56\%, $95 \%$ CI 49, 62). The children were also asked to rate each intervention item from the kit bags on a 5-point scale (love it, like it, don't know, don't like it, hate it); this was re-coded into love/like it and don't know, don't like or hate it. On average, the Project Tomato items that the children liked/loved the most were the fruity face (159/171; 93\%, 95\% CI 89, 97), the Christmas cake recipe (177/218; $81 \%$, 95\% CI 76, 86), the portion game (184/217; $85 \%, 95 \%$ CI 80, 90), and fruit 'n' veg snack box (192/218; $88 \%, 95 \%$ CI 84, 92). Pearson's product-moment correlation coefficient was used to test the relationship between appreciation of the intervention items and implementation. The results revealed that there was a moderate association between implementation and appreciation. Children and parents who liked the different intervention items tended to use them more (children: $r=0.58, n 95, P<0 \cdot 01$; parents: $r=0 \cdot 39, n 57, P<0 \cdot 1)$.

\section{Intervention implementation and the association witb total fruit and vegetable intake}

A two-level regression model was conducted to explore the relationship between children's total follow-up fruit and vegetable intake and overall implementation of school items. The unadjusted and adjusted (gender, ethnicity, baseline intake and IMD score) models are presented in Table 3.

Regression models revealed that for total implementation of school items (curriculum lessons and tasting sessions) there was no significant difference between intervention implementation levels and follow-up fruit and vegetable intake in the control and intervention groups, after adjusting for baseline fruit and vegetable intake, gender, ethnicity and IMD score. While parents' level of implementation was not significantly associated with improvements in fruit and vegetable consumption, after adjusting for possible confounders, parents with a medium implementation of the intervention items had an increase of $40.8(95 \% \mathrm{CI}-67 \cdot 9,149 \cdot 5) \mathrm{g}$ more fruit and vegetables, and parents with a high implementation level had a $66 \cdot 1(95 \% \mathrm{CI}-71 \cdot 4,203 \cdot 7) \mathrm{g}$ increase in fruit and vegetables compared with parents who had a low implementation rating. This suggests that children of parents who had a high or medium intervention implementation consumed more fruit and vegetables compared with those whose parents had a low level of implementation, although it was not statistically significant.

\section{Intervention appreciation and the association with total fruit and vegetable intake}

Table 3 also shows that although the appreciation of various aspects of the project was high, it was not significantly associated with change in children's fruit and vegetable intake. The results did suggest a trend in fruit and vegetable intake with teacher's appreciation of tasting sessions, with an average increase of $38.5(95 \% \mathrm{CI}-6 \cdot 7,83 \cdot 7) \mathrm{g}$ in fruit 
Table 3 Impact on fruit and vegetable intake at follow-up, according to degree of implementation and appreciation of the Project Tomato intervention

\begin{tabular}{|c|c|c|c|c|c|c|c|}
\hline \multirow[b]{2}{*}{ Degree of implementation } & \multicolumn{3}{|c|}{ Unadjusted model } & \multicolumn{3}{|c|}{ Adjusted modelt } & \multirow[b]{2}{*}{$n$} \\
\hline & $\begin{array}{l}\text { Fruit \& vegetable } \\
\text { intake }(\mathrm{g})\end{array}$ & $95 \% \mathrm{Cl}$ & $P$ value & $\begin{array}{l}\text { Fruit \& vegetable } \\
\text { intake }(\mathrm{g})\end{array}$ & $95 \% \mathrm{Cl}$ & $P$ value & \\
\hline \multicolumn{8}{|l|}{ Teachers } \\
\hline Curriculum lessons & $-0 \cdot 8$ & $-34 \cdot 4,32 \cdot 7$ & 0.95 & $-11 \cdot 5$ & $-50 \cdot 1,26 \cdot 9$ & 0.54 & 274 \\
\hline Tasting sessions & $3 \cdot 6$ & $-32 \cdot 4,39 \cdot 5$ & $0 \cdot 83$ & $-12 \cdot 3$ & $-50 \cdot 1,25 \cdot 5$ & 0.50 & 274 \\
\hline \multicolumn{8}{|l|}{ Children } \\
\hline Total implementation & $0 \cdot 9$ & $-0 \cdot 4,2 \cdot 1$ & $0 \cdot 16$ & $1 \cdot 1$ & $-0 \cdot 2,2 \cdot 4$ & $0 \cdot 08$ & 115 \\
\hline \multicolumn{8}{|l|}{ Parents } \\
\hline Medium & $48 \cdot 6$ & $-56 \cdot 4,153 \cdot 7$ & & $40 \cdot 8$ & $-67 \cdot 9,149 \cdot 5$ & & \\
\hline High & $56 \cdot 4$ & $-35 \cdot 4,208 \cdot 3$ & $0 \cdot 34$ & $66 \cdot 1$ & $-71 \cdot 4,203 \cdot 7$ & 0.58 & 57 \\
\hline \multicolumn{8}{|l|}{ Teachers } \\
\hline Curriculum lessons & $2 \cdot 7$ & $-85 \cdot 8,91 \cdot 3$ & 0.94 & $26 \cdot 2$ & $-27 \cdot 2,79 \cdot 6$ & $0 \cdot 26$ & 75 \\
\hline Tasting sessions & $38 \cdot 5$ & $-6 \cdot 7,83 \cdot 7$ & 0.08 & $33 \cdot 1$ & $-24 \cdot 5,90 \cdot 7$ & $0 \cdot 23$ & 145 \\
\hline \multicolumn{8}{|l|}{ Children } \\
\hline Appreciation score & $1 \cdot 5$ & $-0 \cdot 6,4 \cdot 6$ & $0 \cdot 31$ & $1 \cdot 0$ & $-1 \cdot 4,3 \cdot 4$ & $0 \cdot 38$ & 101 \\
\hline \multirow{2}{*}{\multicolumn{8}{|c|}{$\begin{array}{l}\text { Parents } \\
\text { Appreciation score }\end{array}$}} \\
\hline & & & & & & & \\
\hline Medium & $-32 \cdot 4$ & $-112 \cdot 3,47 \cdot 6$ & & $-17 \cdot 7$ & $-80 \cdot 5,45 \cdot 0$ & & \\
\hline High & $-28 \cdot 7$ & $-84 \cdot 5,27 \cdot 1$ & 0.53 & $-39 \cdot 3$ & $-97 \cdot 4,18 \cdot 7$ & $0 \cdot 36$ & 112 \\
\hline
\end{tabular}

tAdjusted for baseline fruit and vegetable intake, gender, Index of Multiple Deprivation score and ethnicity.

and vegetables in the unadjusted model and by $33 \cdot 1$ (95\% CI $-24 \cdot 5,90 \cdot 7) \mathrm{g}$ of fruit and vegetables in the adjusted model. However these differences were not significant.

\section{Otber mechanisms associated with total fruit and vegetable intake}

Parents were also asked 'How many days per school week do you send your child to school with fruit or vegetables?' After adjusting for baseline fruit and vegetable intake, gender, ethnicity and IMD score, there was no significant difference in how many days per week children were sent to school with a piece of fruit or vegetable and their overall fruit and vegetable intake ( $n$ 111; $2-3 \mathrm{~d} /$ week: $-6 \cdot 1$ (95\% CI $-104 \cdot 9,92 \cdot 7)$ g; 4-5 d/week: 23.9 (95\% CI $25 \cdot 0,72 \cdot 3) \mathrm{g} ; P=0 \cdot 3)$. Parents were also asked 'How many nights a week do you eat an evening meal with your child, at a table?' The multilevel regression model revealed that there was a significant relationship between eating at a table and children's fruit and vegetable intake. Children who ate their evening meal at a table 3-7 nights/week with their parents ( $n$ 102) consumed on average 37.6 (95\% CI $9 \cdot 8$, 65.4) g more fruit and vegetables $(P=0 \cdot 01)$ than children who ate their evening meals with their parents $0-2$ nights/ week, after adjusting for sex, ethnicity, IMD score, and baseline fruit and vegetable intake.

\section{Discussion}

The evaluation of the impact of the Project Tomato intervention indicated that a flexible multi-component intervention had little effect on maintaining or increasing children's fruit and vegetable intake in the UK. The components designed and used in this programme are similar to those used in other multi-component interventions which have been shown to be effective ${ }^{(5,10,14,17,19,22)}$. However, implementation rate is a vital component in the success of these studies. In a European study, Te Velde et $a l^{(17)}$ achieved long-term change in children's fruit and vegetable intake in the country that had the highest implementation rate. Similarly, a successful intervention reported by Story et al. ${ }^{(23)}$ recorded high adherence to the intervention materials in their process evaluation.

The implementation of the present intervention for teachers, parents and children was low. However, children with parents who were highly involved in the programme had higher fruit and vegetable intake. This implies that the effect of the intervention on the main outcome, fruit and vegetable intake, might have improved with stronger overall implementation. These results are similar to previous studies $^{(11,18)}$ which had low implementation levels and reported the primary barrier to teaching the intervention was preparation time. Bere et al. $^{(18)}$ had low implementation across the schools due to the intervention materials needing too much preparation time, which resulted in little difference in fruit and vegetable intake.

For the core school materials the teachers' total implementation was particularly low, with less than half the curriculum lessons and on average a quarter of the tasting session intervention items being implemented. The customised school items such as cooking and growing club materials were also poorly implemented, with each school on average only implementing $8 \%$ of the intervention. While the Project Tomato schools were offered funding for cooking and growing clubs, and received the necessary funding and equipment to prepare the tasting sessions, these activities are preparation intense. Without extra assistance from a member of staff or parents, the teachers 
seemed reluctant to implement these types of materials. Multi-component interventions are often seen as labour intensive for non-essential curriculum items and if the teacher-parent relationship is poor, or the parents are rarely involved in school activities, barriers may exist to implementing nutritional interventions ${ }^{(7)}$.

The intervention was designed to be pragmatic and not rely on external agencies for its delivery. This was deliberate, since had the intervention been successful the approach would have been readily transferable between schools. However the majority of successful fruit and vegetable interventions have had external assistance in training, delivering and running of the intervention ${ }^{(9,10,12,13)}$. One successful study ${ }^{(9)}$ provided each school with a 'special resource teacher'. These special resource teachers assisted the year group teacher in implementing the intervention items and increasing the nutrition content of their lessons. School teachers have limited time and resources to spend on extracurricular items; furthermore, funding of additional staff adds considerably to the cost of the programme.

Children's and parents' implementation levels of the home items provided a positive association with children's fruit and vegetable intake. While this difference was not statistically significant, children with parents who implemented more intervention items consumed more fruit and vegetables. The analysis indicated the 'fruit and veg portion game' as one of the most used and liked items by children and parents. This activity involves each member of the household recording their daily intake of fruit and vegetables, to see who has eaten the most at the end of the week. It is a very simple concept that could easily be implemented in public health initiatives. The study also identified parents were less likely to implement activities that involved purchasing ingredients, such as the recipes, compared with the activities where all the resources were provided; demonstrating that cost and preparation time are important factors to consider when designing home intervention materials. A low level of implementation of home materials is consistent with existing research ${ }^{(11-13)}$. Some studies ${ }^{(11-13)}$ have found that parents' involvement in the interventions decreased over time.

Teachers, parents and children had high total appreciation scores for the different intervention materials but this did not necessarily lead to increases in implementation of the items. The results identified that there was no association between the teachers', parents' or children's appreciation scores and fruit and vegetable consumption. Nevertheless, the children with teachers who appreciated the tasting sessions had on average a higher fruit and vegetable intake of just over $0 \cdot 4$ of a portion. This was not statistically significant, perhaps due to the fact that only a quarter of schools implemented the tasting sessions, reducing the power of the study.

Other possible mechanisms associated with children's fruit and vegetable intake were explored. The results revealed that children who eat their main meal with their parents at a table at least three times weekly on average consume half a portion more of fruit and vegetables, compared with children who eat together with their parents less often. This suggests that programmes encouraging parents to eat together with their children at a table may be an effective way of increasing fruit and vegetable intake. These findings support previous research that parents' influence is an important determinant of children's diet ${ }^{(5,27)}$. Eating together at a table provides the perfect environment for parents to model good nutritional behaviour ${ }^{(28)}$. Food preparation, such as planning and writing a shopping list, has been associated with higher fruit and vegetable intake from a study of women aged $18-65$ years $^{(29)}$. Also Taveras et $a l^{(30)}$ found that eating a family meal together was inversely associated with obesity in children aged 9 to 14 years. Children need to see adults eating fruit and vegetables, to help demonstrate positive behaviour ${ }^{(6)}$.

There were limitations to the present study. Validity and reliability of the process measures questionnaires have not been tested; however, this is a common weakness with health interventions, as limited resources are allocated to process evaluations ${ }^{(5,13)}$. Teachers, parents and children might be inclined to give socially desirable answers, leading to overestimation of the intervention effect.

Another limitation is that the study is subject to the wellestablished statistical problems of multiple comparisons or testing ${ }^{(31)}$. The study was powered to analyse the main trial outcome, i.e. change in fruit and vegetable intake between children in the intervention and control groups, and as a consequence it may not be adequately powered for the analysis undertaken in this process measures analysis from the intervention group alone. The main strengths of the present study are that it provides information on process measures of the first multi-component intervention trial to promote fruit and vegetables conducted across England and used advanced statistical techniques.

\section{Conclusions}

The Project Tomato intervention was poorly implemented by teachers. The analysis revealed that a classroom teacherled intervention did not have a positive association with children's diets. Future intervention research should design activities that involve external support or professional training for classroom teachers. The present study also confirmed the importance of parents' involvement and the home environment. Further research should be conducted to review family eating behaviours and the mealtime environment to facilitate change in children's dietary behaviours.

\section{Acknowledgements}

The project was funded by the National Prevention Research Initiative (NPRI), Medical Research Council Registry code G0501297. The authors have no conflicts 
of interest. M.S.C. was involved in designing the intervention, developing materials and analysing the feedback data. C.E.L.E. was involved statistical analysis of the main trial. J.K.R. was involved in coordinating the day-to-day management of the project. D.C.G. was involved in the grant application and statistical analysis of the main trial. J.D.T. was the database manager. J.E.C. was the principal investigator. The authors thank Jennifer Jupp, Christine Webster, Frances Reed, Janice Walker and Ben Styles at the National Foundation for Educational Research (NFER) for their work in sample selection, liaising with the schools and in the collection, entry and distribution of the data.

\section{References}

1. Wells L \& Nelson M (2005) The National School Fruit Scheme produces short-term but not longer-term increases in fruit consumption in primary school children. Br J Nutr 93, 537-542.

2. Ransley JK, Greenwood DC, Cade JE et al. (2007) Does the school fruit and vegetable scheme improve children's diet? A non-randomised controlled trial. J Epidemiol Community Health 61, 699-703.

3. Fogarty AW, Antoniak M, Venn AJ et al. (2007) Does participation in a population-based dietary intervention scheme have a lasting impact on fruit intake in young children? Int J Epidemiol 36, 1080-1085.

4. Fisher JO, Mitchell DC, Smiciklas-Wright H et al. (2002) Parental influences on young girls' fruit and vegetable, micronutrient, and fat intakes. J Am Diet Assoc 102, 58-64.

5. Wind M, Bjelland M, Perez-Rodrigo C et al. (2008) Appreciation and implementation of a school-based intervention are associated with changes in fruit and vegetable intake in 10- to 13-year old schoolchildren - the Pro Children study. Health Educ Res 23, 997-1007.

6. French SA \& Stables G (2003) Environmental interventions to promote vegetable and fruit consumption among youth in school settings. Prev Med 37, 593-610.

7. Knai C, Pomerleau J, Lock K et al. (2006) Getting children to eat more fruit and vegetables: a systematic review. Prev Med 42, 85-95.

8. Ciliska D, Miles E, O'Brien MA et al. (2000) Effectiveness of community-based interventions to increase fruit and vegetable consumption. J Nutr Educ 32, 341-352.

9. Auld G, Romaniello C, Heimendinger J et al. (1998) Outcomes from a school-based nutrition education program using resource teachers and cross-disciplinary models. J Nutr Educ 30, 268-280.

10. Perry CL, Bishop DB, Taylor G et al. (1998) Changing fruit and vegetable consumption among children: the 5-a-Day Power Plus program in St. Paul, Minnesota. Am J Public Health 88, 603-609.

11. Baranowski J, Doyle C, Lin LS et al. (2000) Gimme 5 fruit, juice, and vegetables for fun and health: outcome evaluation. Health Educ Behav 27, 96-111.

12. Reynolds KD, Franklin FA, Binkley D et al. (2000) Increasing the fruit and vegetable consumption of fourth-graders: results from the high 5 project. Prev Med 30, 309-319.

13. Sahota P, Rudolf MC, Dixey R et al. (2001) Randomised controlled trial of primary school based intervention to reduce risk factors for obesity. BMJ 323, 1029-1032.
14. Perry CL, Bishop DB, Taylor GL et al. (2004) A randomized school trial of environmental strategies to encourage fruit and vegetable consumption among children. Health Educ Behav 31, 65-76.

15. Cullen KW, Watson K, Baranowski T et al. (2005) Squire's Quest: intervention changes occurred at lunch and snack meals. Appetite 45, 148-151.

16. Bere E, Veierod MB \& Klepp K-I (2005) The Norwegian School Fruit Programme: evaluating paid vs. no-cost subscriptions. Prev Med 41, 463-470.

17. Te Velde SJ, Brug J, Wind $M$ et al. (2008) Effects of a comprehensive fruit- and vegetable-promoting schoolbased intervention in three European countries: the Pro Children Study. Br J Nutr 99, 893-903.

18. Bere E, Veierod MB, Bielland $M$ et al. (2006) Outcome and process evaluation of a Norwegian school-randomized fruit and vegetable intervention: Fruits and Vegetables Make the Marks (FVMM). Health Educ Res 21, 258-267.

19. Tak NI, Te Velde SJ \& Brug J (2007) Ethnic differences in 1-year follow-up effect of the Dutch Schoolgruiten Project - promoting fruit and vegetable consumption among primary-school children. Public Health Nutr 10, 1497-1507.

20. Kitchen MS, Ransley JK, Greenwood DC et al. (2009) Study protocol: a cluster randomised controlled trial of a school based fruit and vegetable intervention - Project Tomato. BMC Health Serv Res 9, 101.

21. Baranowski T, Anderson C \& Carmack C (1998) Mediating variables framework in physical activity interventions: How are we doing? How might we do better? Am J Prev Med 15, 266-297.

22. Steckler A \& Linnan L (2002) Process Evaluation for Public Health Interventions and Research. San Francsico, CA: Jossey-Bass.

23. Story M, Mays RW, Bishop DB et al. (2000) 5-a-day Power Plus: process evaluation of a multicomponent elementary school program to increase fruit and vegetable consumption. Health Educ Behav 27, 187-200.

24. Conner M \& Sparks P (2005) The theory of planned behaviour and health behaviour. In Predicting Health Behaviour, pp. 170-222 [M Conner and P Norman, editors]. Buckingham: Open University Press.

25. Cade JE, Frear L \& Greenwood DC (2006) Assessment of diet in young children with an emphasis on fruit and vegetable intake: using CADET - Child and Diet Evaluation Tool. Public Health Nutr 9, 501-508.

26. Noble M, Wright G, Dibben C et al. (2004) The English Indices of Deprivation 2004: Report to the Office of the Deputy Prime Minister. London: Neighbourhood Renewal Unit.

27. Blanchette L \& Brug J (2005) Determinants of fruit and vegetable consumption among 6-12-year-old children and effective interventions to increase consumption. I Hum Nutr Diet 18, 431-443.

28. Patrick H, Nicklas TA, Patrick H et al. (2005) A review of family and social determinants of children's eating patterns and diet quality. J Am Coll Nutr 24, 83-92.

29. Crawford D, Ball K, Mishra G et al. (2007) Which foodrelated behaviours are associated with healthier intakes of fruits and vegetables among women? Public Health Nutr 10, 256-265.

30. Taveras EM, Rifas-Shiman SL, Berkey CS et al. (2005) Family dinner and adolescent overweight. Obes Res 13, 900-906.

31. Miller RG (1981) Simultaneous Statistical Inference. New York: Springer Verlag. 